\title{
Decentralized Coordinated Fuzzy Stabilization Control of Power System: An LMI Approach
}

\author{
Shihe $\mathrm{CHEN}^{1}$, Xi ZHANG ${ }^{1}$, Xiaoming $\mathrm{LI}^{2 \mathrm{a}}$, Shilin WANG ${ }^{2}$, Yan $\mathrm{PAN}^{2}$, Yuguang \\ NIU $^{2}$, Zhongwei LIN ${ }^{2}$ \\ ${ }^{1}$ Electric Power Research Institute of Guangdong Power Grid Corporation, Guangzhou, 510000, \\ China
}
${ }^{2}$ School of control \& computer engineering, North China Electric power University, Beijing, 102206, China
${ }^{a}$ E-mail: lixiaoming@ncepu.edu.cn

\begin{abstract}
Keywords: power system; decentralized coordinated control; T-S fuzzy model; stabilization control; linear matrix inequality
\end{abstract}

\begin{abstract}
This paper introduces a decentralized coordinated fuzzy stabilization control of power system. First, the Takagi and Sugeno (T-S) fuzzy model is employed to approximate system-wide nonlinear model of power system. Next, based on the fuzzy model, a fuzzy controller with decentralized constrain is developed to achieve the suboptimal control. A robust stabilization technique is also proposed to override the oscillations. Finally, the suboptimal control is parameterized in terms of three eigenvalue problems (EVP's), which can be solved conveniently by using linear matrix inequality (LMI) optimization techniques. A simulation example is used to illustrate the contributions of the proposed method. The results demonstrate that the proposed method gives a desired performance.
\end{abstract}

\section{Introduction}

Power system is large-scale, interconnected, nonlinear and geographical extensive. The controller design of power system is commonly based on decentralized principle [1], which is only depended on the local or subsystem based signals. In the early stage of power system, the interactions between the different subsystems have not been considered for the simple structure and lack of understanding of power system. Therefore, all the controllers were separate controller based on the separate model in that period. Obviously, this separate decentralized controller only had certain effect on installed local system, which can not provide certain effects on the neighboring systems. In a particular case, the system-wide performance maybe worsen for the chaos between those separate decentralized controllers. It can be concluded that the stability of power system must be considered from all involving relations globally in the transient control [2-4].

Decentralized coordinated control of power system has been widely reported in the past [5-12]. The decentralized coordinated control strategy proposed by Geromel and Bernussou [5] is more feasible, which is designed based on the integrated, interaction-considered and system-wide model. Under the decentralized constrains, local controllers are coordinated to minimize the system-wide performance effectively. Based on this, a decentralized feedback stabilization method for a large-scale, interconnected system has been proposed in [6]. Furthermore, the decentralized coordinated control method with output feedback has been introduced by Yingduo HAN et al. [7]. However, a terms of problems of output feedback decentralized coordinated control of power system have been solved, such as system-wide model and control algorithm [8], coordinated effect of weight matrix and optimal weight matrix calculation [9], chose and combination of feedback variables [10], and installed location of decentralized coordinated controller [11]. Those mentioned methods provide a systematic solution for the decentralized coordinated control of power system, where the Livine-Athans (L-A) equations are needed to be calculated in those methods. However, the L-A equations are not easy to be solved, especially for cross-coupled problems. As a solution, Khargonekar et al. give an algorithmic solution to the optimal control problem for linear systems 
based on a convex optimization approach [12]. More recently, LMI techniques have been employed to deal with those cross-coupled problems.

In this paper, a T-S fuzzy model based decentralized coordinated control of power system is proposed, where a robust technical is employed to stabilize the system. A simulation example is provided to illustrate the contributions of the proposed method. The results show that the desired performances can be achieved. The paper is organized as follows. The controller design is presented in Section II. In Section III, a simulation example is provided to demonstrate the contributions. Finally, conclusions are drawn in Section IV.

\section{CONTROLLER DESIGN}

The system-wide linearized state equation for a power system with $N$ generators can be written as following form, where the interactions have been considered.

$$
\dot{X}(t)=A X(t)+B U(t)
$$

Here $X(\mathrm{t})=\left[x_{1}(\mathrm{t}), \ldots, x_{N}(\mathrm{t})\right]^{\mathrm{T}}$ is the state vector, and $U=\left[u_{1}(\mathrm{t}), \ldots, u_{N}(\mathrm{t})\right]^{\mathrm{T}}$ is the manipulated vector. The details about those variables included matrixes $A$ and $B$, are given in [8]. The T-S fuzzy model is employed to cope with the nonlinearity of power system, the TS fuzzy rule is presented as:

\section{Plant Rule $i$}

If $z_{1}(\mathrm{t})$ is $F_{i 1}$, and $\ldots$ and $z_{\mathrm{g}}(\mathrm{t})$ is $F_{i g}$

Then $\dot{X}(t)=A_{i} X(t)+B_{i} U(t),(i=1, \ldots, L)$

Where $F_{\mathrm{ij}}$ is the fuzzy set, $L$ is the number of if-then rules, and $z_{1}(\mathrm{t}) \ldots z_{\mathrm{g}}(\mathrm{t})$ are the premise variables. Then, the final output of the fuzzy system is inferred as follows:

$$
\dot{X}(t)=\sum_{i=1}^{L} h_{i}(t)\left\{A_{i} X(t)+B_{i} U(t)\right\}
$$

Where $z(t)=\left[\begin{array}{lll}z_{i}(t) & \ldots & z_{g}(t)\end{array}\right], \quad \mu_{i}(z(t))=\prod_{j=1}^{L} F_{i j}\left(z_{j}(t)\right), h_{i}(t)=\mu_{i}(z(t)) / \sum_{i=1}^{L} \mu_{i}(z(t))$, and $F_{i j}\left(z_{j}(t)\right)$ is the grade of membership of $z_{j}(t)$ in $F_{i j}$.

In this paper, it is assumed that $\mu_{i}(z(t)) \geq 0$ and $\sum_{i=1}^{L} \mu_{i}(z(t))>0$, therefore, $h_{i}(t) \geq 0$ and $\sum_{i=1}^{L} h_{i}(t)=1$.For achieving decentralized control, each local controller should be only depended on the local signals.

\section{Controller Rule $j$}

If $z_{1}(\mathrm{t})$ is $F_{j 1}$, and $\ldots$ and $z_{\mathrm{g}}(\mathrm{t})$ is $F_{j g}$

Then $U_{j}(t)=K_{d j} X(t),(j=1, \ldots, L)$

Hence, the fuzzy controller is given by

$$
U(t)=\sum_{j=1}^{L} h_{j}(t) K_{d j} X(t)
$$

Where $K_{d j}=b l k\left\{K_{d 1 \mathrm{j}}, \ldots, K_{d N j}\right\}$ is a block diagonal matrix, $K_{d j}$ (for $j=1,2, \ldots, L$ ) are the control parameters.

The following performance is widely used in the design of excitation controller of synchronous generator (SG).

$$
J(U)=\int_{t_{0}}^{t_{f}}\left\{X^{\mathrm{T}}(t) Q X(t)+U^{\mathrm{T}}(t) R U(t)\right\} d t
$$

Before solving (6), the stability of the closed-loop system in (3) must be guaranteed at the equilibrium $X(\mathrm{t})=0$. The chosen Lyapunov function is

$V(t)=X^{\mathrm{T}}(t) P X(t)$

Where $P$ is a positive matrix. By differentiating (7), 


$$
\begin{aligned}
& \begin{aligned}
\dot{V}(t) & =\dot{X}^{\mathrm{T}}(t) P X(t)+X^{\mathrm{T}}(t) P \dot{X}(t) \\
& =\sum_{i=1}^{L} h_{i}(t) \sum_{j=1}^{L} h_{j}(t)\left(\left(A_{i}+B_{i} K_{d j}\right) X(t)\right)^{\mathrm{T}} P X(t)+X^{\mathrm{T}}(t) P\left(A_{i}+B_{i} K_{d j}\right) X(t) \\
& =\sum_{i=1}^{L} h_{i}(t) \sum_{j=1}^{L} h_{j}(t) X^{\mathrm{T}}(t)\left\{\left(A_{i}+B_{i} K_{d j}\right)^{\mathrm{T}} P+P\left(A_{i}+B_{i} K_{d j}\right)\right\} X(t)
\end{aligned} \\
& \text { If }\left(A_{i}+B_{i} K_{d j}\right)^{\mathrm{T}} P+P\left(A_{i}+B_{i} K_{d j}\right)<0 \\
& \text { Then } \quad \dot{V}(t)<0 .
\end{aligned}
$$

This shows that the closed-loop system in (3) is locally quadratically stable at the equilibrium $X(\mathrm{t})=0$ under the constrains of (9). For that the feedback gain matrix of $K_{d}$ is strictly confined to be a block diagonal matrix, it is difficult to obtain the optimal solution. In this situation, a sub-optimal method is adopted to minimize the upper bound of $J(U)$. Substituting (5) into (6),

$$
\begin{aligned}
J(U)= & \int_{0}^{t_{f}}\left\{X^{T}(t) Q X(t)+\left(\sum_{j=1}^{L} h_{j}(t) K_{d j} X(t)\right)^{\mathrm{T}} \times R \sum_{j=1}^{L} h_{j}(t) K_{d j} X(t)\right\} d t \\
= & X^{\mathrm{T}}(0) P X(0)-X^{\mathrm{T}}\left(t_{f}\right) P X\left(t_{f}\right)+\int_{0}^{t_{f}}\left\{X^{\mathrm{T}}(t) Q X(t)\right. \\
& \left.+\left(\sum_{j=1}^{L} h_{j}(t) K_{d j} X(t)\right)^{\mathrm{T}} R\left(\sum_{j=1}^{L} h_{j}(t) K_{d j} X(t)\right)+\frac{d}{d t}\left(X^{\mathrm{T}}(t) P X(t)\right)\right\} d t \\
\leq & X^{\mathrm{T}}(0) P X(0)+\int_{0}^{t_{f}}\left\{X^{\mathrm{T}}(t)\left(Q+\sum_{j=1}^{L} h_{j}(t) K_{d j}^{\mathrm{T}} R K_{d j}\right)\right. \\
& \left.\times X(t)+\dot{X}^{\mathrm{T}}(t) P X(t)+X^{\mathrm{T}}(t) P \dot{X}(t)\right\} d t \\
\leq & X^{\mathrm{T}}(0) P X(0)+\int_{0}^{t_{f}} \sum_{i=1}^{L} h_{i}(t) \sum_{j=1}^{L} h_{j}(t)\left\{X^{\mathrm{T}}(t)(Q\right. \\
& \left.\left.+K_{d j}^{\mathrm{T}} R K_{d j}+\left(A_{i}+B_{i} K_{d j}\right)^{\mathrm{T}} P+P\left(A_{i}+B_{i} K_{d j}\right)\right) X(t)\right\} d t \\
\text { If }\left(A_{i}+\right. & \left.B_{i} K_{d j}\right)^{\mathrm{T}} P+P\left(A_{i}+B_{i} K_{d j}\right)+K_{d j}^{\mathrm{T}} R K_{d j}+Q<0
\end{aligned}
$$

Then upper bound of $J(U)$ is optimized.

$$
J(U) \leq X^{\mathrm{T}}(0) P X(0)
$$

From the analysis above, the decentralized coordinated control of power system can be formulated as the following optimization problem.

$$
\min _{P}: X^{\mathrm{T}}(0) P X(0)
$$

Subject to: $\quad P=P^{\mathrm{T}}>0$, (9) and (11)

In order to give a detailed example of LMI based solution, a three-machine system is introduced here. The solution for the systems with more than three machines follows a similar approach.

For simplifying the solution, the $P=b l k\left\{P_{1}, P_{2}, P_{3}\right\}, Q=b l k\left\{Q_{1}, Q_{2}, Q_{3}\right\}$ and $R=b l k\left\{R_{1}, R_{2}, R_{3}\right\}$ are predefined as the block diagonal matrixes. Matrix $B=b l k\left\{B_{1 \mathrm{i}}, B_{2 \mathrm{i}}, B_{3 \mathrm{i}}\right\}_{i,=1, \ldots, L}$ must be a block diagonal matrix too. Expanding (9) and (11),

$$
\left[\begin{array}{ccc}
\left(A_{11 i}+B_{1 i} K_{d 1 j}\right)^{\mathrm{T}} P_{1} & A_{21 i}^{\mathrm{T}} P_{2}+P_{1} A_{12 i} & A_{31 i}^{\mathrm{T}} P_{3}+P_{1} A_{13 i} \\
+P_{1}\left(A_{11 i}+B_{1 i} K_{d 1 j}\right) & & \\
A_{12 i}^{\mathrm{T}} P_{1}+P_{2} A_{21 i} & \left(A_{22 i}+B_{2 i} K_{d 2 j}\right)^{\mathrm{T}} P_{2} & A_{32 i}^{\mathrm{T}} P_{3}+P_{2} A_{23 i} \\
& +P_{2}\left(A_{22 i}+B_{2} K_{d 2 j}\right) & \\
A_{13 i}^{\mathrm{T}} P_{1}+P_{3} A_{31 i} & A_{23 i}^{\mathrm{T}} P_{2}+P_{3} A_{32 i} & \left(A_{33 i}+B_{3} K_{d 3 j}\right)^{\mathrm{T}} P_{3} \\
& & +P_{3}\left(A_{33 i}+B_{3 i} K_{d 3 j}\right)
\end{array}\right]<0
$$




$$
\left[\begin{array}{ccc}
\left(A_{11 i}+B_{1 i} K_{d 1 j}\right)^{\mathrm{T}} P_{1} & \\
+P_{1}\left(A_{11 i}+B_{1 i} K_{d 1 j}\right) & A_{21 i}^{\mathrm{T}} P_{2}+P_{1} A_{12 i} & A_{31 i}^{\mathrm{T}} P_{3}+P_{1} A_{13 i} \\
+K_{d 1 j}^{\mathrm{T}} R_{1} K_{d 1 j}+Q_{1} & & \\
& \left(A_{22 i}+B_{2 i} K_{d 2 j}\right)^{\mathrm{T}} P_{2} & \\
A_{12 i}^{\mathrm{T}} P_{1}+P_{2} A_{21 i} & +P_{2}\left(A_{22 i}+B_{2 i} K_{d 2 j}\right) & A_{32 i}^{\mathrm{T}} P_{3}+P_{2} A_{23 i} \\
& +K_{d 2 j}^{\mathrm{T}} R_{2} K_{d 2 j}+Q_{2} & \\
& & \left(A_{33 i}+B_{3 i} K_{d 3 j}\right)^{\mathrm{T}} P_{3} \\
A_{13 i}^{\mathrm{T}} P_{1}+P_{3} A_{31 i} & A_{23 i}^{\mathrm{T}} P_{2}+P_{3} A_{32 i} & +P_{3}\left(A_{33 i}+B_{3 i} K_{d 3 j}\right) \\
& & +K_{d 3 j}^{\mathrm{T}} R_{3} K_{d 3 j}+Q_{3}
\end{array}\right]<0
$$

Where matrix $A_{i}=\left\{A_{k g i}\right\}_{i,=1, \ldots, L}$ is a block matrix, $A_{k k i}$ for $k=1,2,3$, is the state matrix of the $k$-th SG and $A_{k g i}$ for $k, g=1,2,3$, and $k \neq g$ represents the interaction matrix between the $k$-th and $g$-th SGs. Each $A_{k g i}$ has its appropriate dimension. Defining $W=W^{\mathrm{T}}=b l k\left\{\begin{array}{lll}P_{1}^{-1} & P_{2}^{-1} & P_{3}^{-1}\end{array}\right\}$ and multiplying it into (15) and (16),

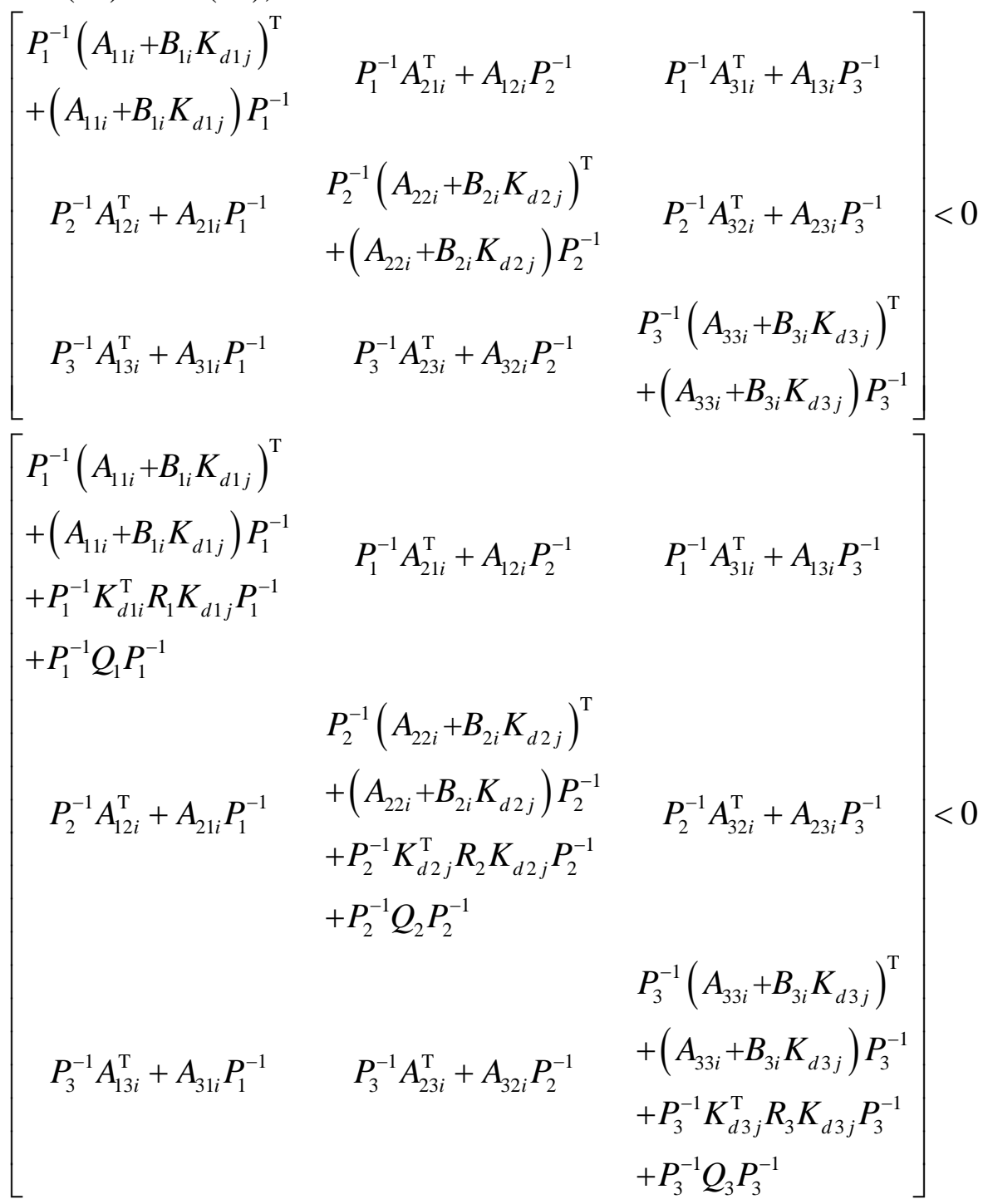

With $Z_{1}=K_{d 1} P_{1}^{-1}, Z_{2}=K_{d 2} P_{2}^{-1}$ and $Z_{3}=K_{d 3} P_{3}^{-1}$, and by the Schur complements [13], the matrix inequalities (17) and (18) can be rearranged as the following forms: 


$$
\begin{aligned}
& {\left[\begin{array}{ccc}
P_{1}^{-1} A_{11 i}^{\mathrm{T}}+Z_{1 j}^{\mathrm{T}} B_{1 i}^{\mathrm{T}} & P_{1}^{-1} A_{21 i}^{\mathrm{T}}+A_{12 i} P_{2}^{-1} & P_{1}^{-1} A_{31 i}^{\mathrm{T}}+A_{13 i} P_{3}^{-1} \\
+A_{11 i} P_{1}^{-1}+B_{1 i} Z_{1 j} & & \\
P_{2}^{-1} A_{12 i}^{\mathrm{T}}+A_{21 i} P_{1}^{-1} & P_{2}^{-1} A_{22 i}^{\mathrm{T}}+Z_{2 j}^{\mathrm{T}} B_{2 i}^{\mathrm{T}} & P_{2}^{-1} A_{32 i}^{\mathrm{T}}+A_{23 i} P_{3}^{-1} \\
& +A_{22 i} P_{2}^{-1}+B_{2 i} Z_{2 j} & \\
P_{3}^{-1} A_{13 i}^{\mathrm{T}}+A_{31 i} P_{1}^{-1} & P_{3}^{-1} A_{23 i}^{\mathrm{T}}+A_{32 i} P_{2}^{-1} & P_{3}^{-1} A_{33 i}^{\mathrm{T}}+Z_{3 j}^{\mathrm{T}} B_{3 i}^{\mathrm{T}} \\
& & A_{33 i} P_{3}^{-1}+B_{3 i} Z_{3 j}
\end{array}\right]<0}
\end{aligned}
$$

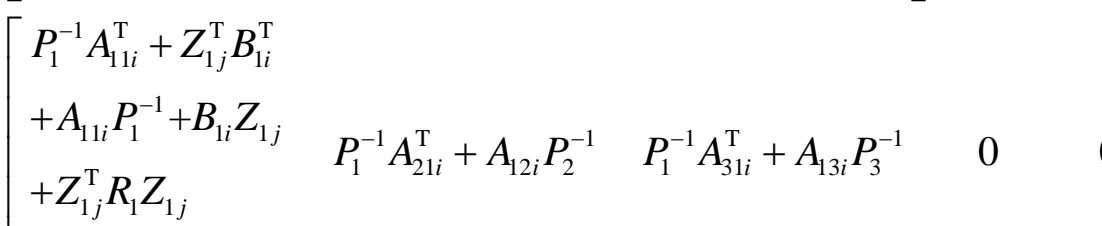

$$
\begin{aligned}
& +P_{1}^{-1} Q_{1} P_{1}^{-1}
\end{aligned}
$$

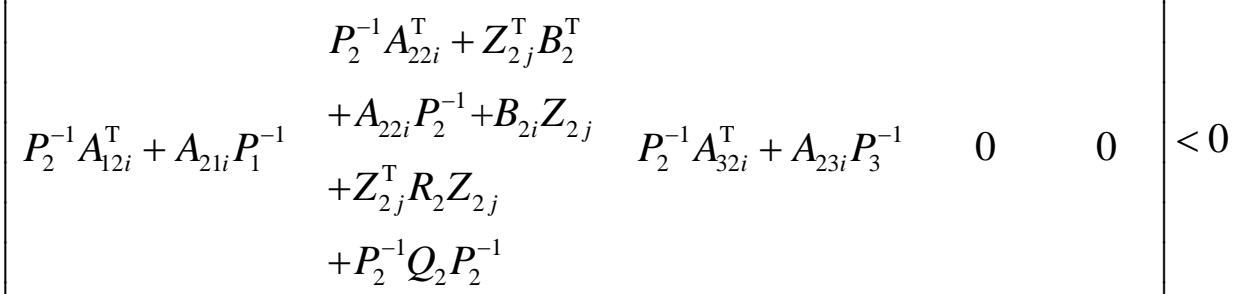

$$
\begin{aligned}
& {\left[\begin{array}{ccccc}
P_{3}^{-1} A_{13 i}^{\mathrm{T}}+A_{31 i} P_{1}^{-1} & P_{3}^{-1} A_{23 i}^{\mathrm{T}}+A_{32 i} P_{2}^{-1} & P_{3}^{-1} A_{33 i}^{\mathrm{T}}+Z_{3 j}^{\mathrm{T}} B_{3}^{\mathrm{T}} & Z_{3 j}^{\mathrm{T}} & 0 \\
0 & 0 & A_{33 i} P_{3}^{-1}+B_{3 i} Z_{3 j} & & \\
0 & 0 & Z_{3 j}^{\mathrm{T}} & -R_{3}^{-1} & P_{3}^{-1} \\
0 & 0 & 0 & P_{3}^{-1} & -Q_{3}^{-1}
\end{array}\right]}
\end{aligned}
$$

Note that (19) and (20) implies that

$$
\begin{aligned}
& P_{2}^{-1} A_{22 i}^{\mathrm{T}}+Z_{2 j}^{\mathrm{T}} B_{2 i}^{\mathrm{T}}+A_{22 i} P_{2}^{-1}+B_{2 i} Z_{2 j}<0 \\
& P_{2}^{-1} A_{22 i}^{\mathrm{T}}+Z_{2 j}^{\mathrm{T}} B_{2 i}^{\mathrm{T}}+A_{22 i} P_{2}^{-1}+B_{2 i} Z_{2 j}+Z_{2 j}^{\mathrm{T}} R_{2} Z_{2 j}+P_{2}^{-1} Q_{2} P_{2}^{-1}<0
\end{aligned}
$$

However, the (22) is still not convex, which can be transformed into the following LMI by using the Schur complements.

$$
\left[\begin{array}{ccc}
P_{2}^{-1} A_{22 i}^{\mathrm{T}}+Z_{2 j}^{\mathrm{T}} B_{2 i}^{\mathrm{T}}+A_{22 i} P_{2}^{-1}+B_{2 i} Z_{2 j} & Z_{2 j} & 0 \\
0 & -R_{2}^{-1} & P_{2}^{-1} \\
Z_{2 j}^{\mathrm{T}} & P_{2}^{-1} & -Q_{2}^{-1}
\end{array}\right]<0
$$

\section{Step 1:}

The $Z_{2 j}=K_{d 2 j} P_{2}^{-1}$ and $P_{2}^{-1}$ can be obtained from following EVP, then $K_{d 2 j}=Z_{2 j} P_{2}$ is obtained. $\min _{P_{2}^{-1}}: x_{2}^{\mathrm{T}}(0) P_{2} x_{2}(0)$

Subject to: $P_{2}=P_{2}^{\mathrm{T}}>0$, (21) and (23)

\section{Step 2:}

By using a similar approach, $K_{d 1}$ and $P_{1}^{-1}$ is obtained from following EVP.

$\min _{P_{1}^{-1}}: x_{1}^{\mathrm{T}}(0) P_{1} x_{1}(0)$

Subject to: $P_{1}=P_{1}^{\mathrm{T}}>0$

$$
P_{1}^{-1} A_{11 i}^{\mathrm{T}}+Z_{1 j}^{\mathrm{T}} B_{1 j}^{\mathrm{T}}+A_{11 i} P_{1}^{-1}+B_{1 i} Z_{1 j}<0
$$




$$
\left[\begin{array}{ccc}
P_{1}^{-1} A_{11 i}^{\mathrm{T}}+Z_{1 j}^{\mathrm{T}} B_{1 i}^{\mathrm{T}}+A_{11 i} P_{1}^{-1}+B_{1 i} Z_{1 j} & Z_{1 j} & 0 \\
0 & -R_{1}^{-1} & P_{1}^{-1} \\
Z_{1 j}^{\mathrm{T}} & P_{1}^{-1} & -Q_{1}^{-1}
\end{array}\right]<0
$$

Step 3:

Substituting $K_{d 1 \mathrm{j}}, P_{1}{ }^{-1}, P_{2}{ }^{-1}$ and $K_{d 2 \mathrm{j}}$ into (19) and (20), it can be found that the (19) and (20) are convex now. $K_{d 3 j}$ and $P_{3}{ }^{-1}$ can be solved from following EVP.

$$
\min _{P_{3}^{-1}}: x_{3}^{\mathrm{T}}(0) P_{3} x_{3}(0)
$$

Subject to: $P_{3}=P_{3}^{\mathrm{T}}>0$, (19) and (20)

The three EVP's can be solved conveniently by using MATLAB LMI toolbox. Then, based on the obtained $K_{d 1 j}, K_{d 2 j}$, and $K_{d 3 j}$ for $j=1,2, \ldots, L$, the fuzzy controller can be constructed according the prescribed fuzzy membership functions and fuzzy sets. In this paper, a three-machine system is employed to demonstrate the derivation. However, this method is not limited to three-machine systems, it can be used in the generic forms of power systems.

From the derivation, it is seen that the decentralized fuzzy controller only depends on local signals. The system-wide performance in (6) can be regarded as coordinated mechanism, and global stability is guaranteed by (9). Hence, the developed method can be considered as a decentralized coordinated fuzzy stabilization control (DCFSC).

\section{Dynamic simulations}

A simple generic three-machine system is used to demonstrate the contributions of the proposed DCFSC in the MATLAB/SIMULINK environment. The structure and configuration of the simulation system are displayed in Fig. 1, where SG1 is PV node, SG2 is PQ node, and SG $\mathrm{e}_{\mathrm{e}}$ is swing node, which is an equivalent generator of outside power system.

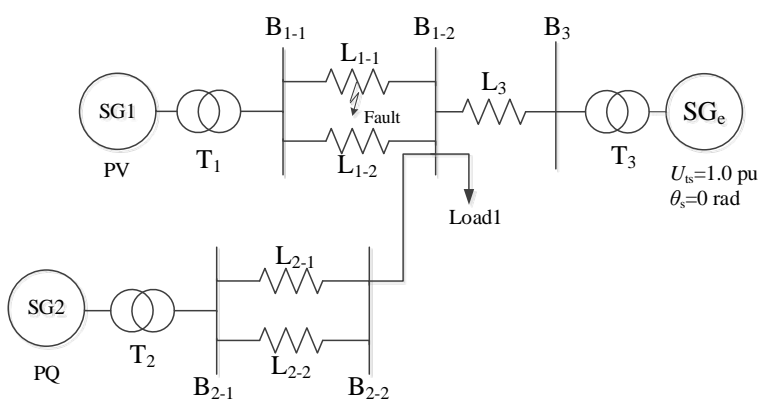

Fig.1 Configuration of simulation system

\section{Dominant eigenvalue analysis}

It is known that system dynamic is mainly determined by the locations of dominant eigenvalues. The dominant eigenvalues of the system in Fig. 1 with different excitation controllers, such as automatic voltage regulator (AVR), power system stabilizer (PSS), and DCFSC, are displayed in Fig. 2.

From Fig. 2, it can be seen that there are two oscillation models when AVR and PSS have been installed. They are $-1.403 \pm \mathrm{j} 5.959$ and $-1.148 \pm \mathrm{j} 5.78$, and the corresponding damping factors are 0.229 and 0.195, respectively. When DCFSC has been installed, the dominant eigenvalues are $-2.156 \pm j 5.6$ and $-2.083 \pm j 6.775$. The dominant eigenvalues are pushed into the left half of the complex plane in comparison with AVR and PSS installed. This means the system damping has been significantly enhanced. For example, the system damping factor with DCFSC is 0.36 for the eigenvalue 2.156 $\pm \mathrm{j} 5.6$.

\section{Response of three- phase ground fault}

In order to investigate the dynamics of the simulation system with different excitation control schemes, a three-phase ground fault was applied in the middle of line L1-1 at $t=0+$, and the fault 
was cleared after 0.15s. The responses of SG1 and SG2 are displayed in Fig. 3 and Fig. 4, respectively, where $P, U_{\mathrm{t}}$ and $\Delta \omega$ denote output active power, terminal voltage, and deviation of

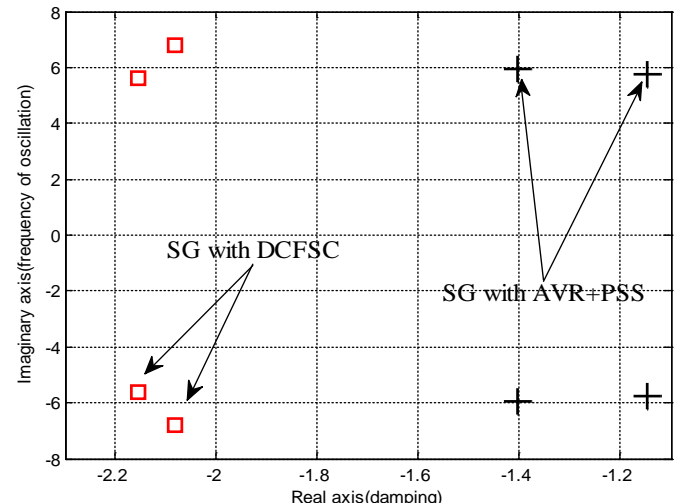

Fig.2 Dominant eigenvalue analysis (SGs with AVR+PSS installed: “+”; SGs with DCFSC installed: “ $\square$ ”)
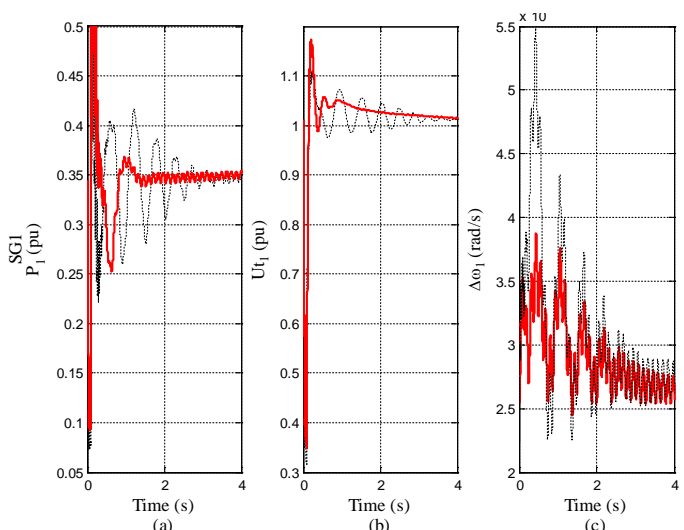

Fig. 3 Three- phase ground fault response of

SG1 (SGs with AVR+PSS installed: dotted line; SGs with DCFSC installed: bold full line)

rotor speed, respectively. The pre- fault condition of the three- phase ground fault is the same as the Fig.2 used.

It can be seen that the transient control performance with the proposed DCFSC is considerable enhanced. It can be found that the system with AVR and PSS became stability approximately at $t=3.5 \mathrm{~s}$ after the fault. However, the system with DCFSC enters stable state at $\mathrm{t}=2 \mathrm{~s}$ after the fault, the transient time is greatly shorten. From the $\Delta \omega$ curves of SG1 and SG2, it can be observed that the capability of resistance to swing is greatly enhanced by using DCFSC.

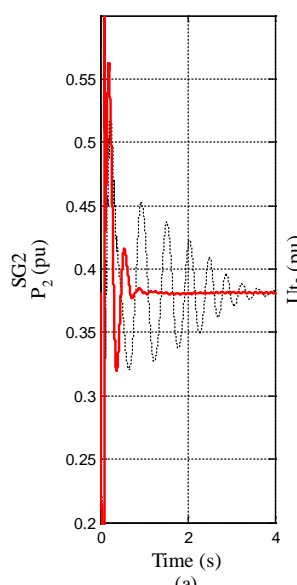

(a)

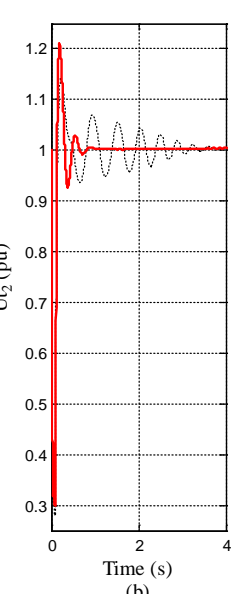

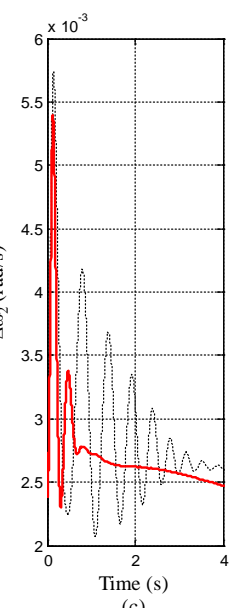

(c)

Fig.4 Three-phase ground fault response of SG2 (SGs with AVR+PSS installed: dotted line;

SGs with DCFSC installed: bold full line)

It is known that only the magnitude of transient electromotive force (EMF) can be manipulated [14]. Namely, there is a strong interaction between the terminal voltage regulation and damping control. The damping improvement is obtained at the cost of terminal voltage control performance. The evidence can be found in Fig. 3 (b) and Fig. 4 (b). The response of the three-phase ground fault conforms the dominant eigenvalue analysis of Fig. 2.

\section{Conclusion}

This paper introduces decentralized coordinated stabilization control of power system, where a T-S fuzzy method is introduced to approximate the nonlinearity of power system. Then, the optimization is transferred into three-EVP's, and each EVP can be described by terms of LMI's, which can be solved conveniently by using MATLAB. The transient benefits and system damping 
with the proposed DCFSC controller are illustrated by both dominant eigenvalue analysis and simulation of time response. This paper shows that LMI has a great capability to solve the cross-coupled problems, which widely exists in the controller designs of power systems.

\section{Acknowledgment}

This work is supported by the National Key Basic Research Program of China (973 Program) under Grant No. 2012CB215203, by the National Nature Science Foundation of China under Grant No. 61203043, and by Fundamental Research Funds for the Central Universities in China.

\section{References}

[1] Yu Y. Electric power system dynamics[J]. ACADEMIC PRESS, INC., 111 FIFTH AVE., NEW YORK, NY 10003, USA, 1983, 256, 1983.

[2] X.X Zhou, J.C. ZHENG, G.R. SHEN, et al. Experience and Lessons learned from blackout in interconnected north america power gird [J]. Power System Technology, 2003, 27(9): 1-1.

[3] Xue-hao HU. Rethinking and enlightenment of large scope blockout in interconnected north america power gird[J]. Power System Technology, 2003, 27(9): J002-J006.

[4] B.S. TANG. Blockout in south of london and its lessons[J] . Power System Technology, 2004, 27(11): 1-5.

[5] Geromel J C, Bernussou J. Optimal decentralized control of dynamic systems[J]. Automatica, 1982, 18(5): 545-557.

[6] Mahalanabis A K, SINGH R. On decentralized feedback stabilization of large-scale interconnected systems[J]. International Journal of Control, 1980, 32(1): 115-126

[7] Y.D HAN, H. J CHEN, Z.H. ZHONG. Optimal decentralized coordinated control of power system[M], Beijing: qinghua university press, 1997.

[8] H. J CHEN, Y.D HAN, Z.H. ZHONG. Study on the decentralized optimal control excitation controllers in multimachine power system[J]. Control theory and applications, , 1989, 6(3): 31-38.

[9] H. J CHEN, Y.D HAN, Z.H. ZHONG. Study on the decentralized output feedback controllers in multimachine power system: part 1, model and algorithm[J]. Proceedings of the CESS, 1990 (2): 32-39.

[10] H. J CHEN, Y.D HAN, Z.H. ZHONG. Study on the decentralized output feedback controllers in multimachine power system: part 2, coordinated seclection of weighting matrix[J]. Proceedings of the CESS, 1990, 10(5): 37-41.

[11] H. J CHEN, Y.D HAN, Z.H. ZHONG. Study on the decentralized output feedback controllers in multimachine power system: part 3, Selection of feedaback variables and siting of controllers[J], Proceedings of the CESS, 1991, 11(2): 23-28.

[12] Khargonekar P. P., Rotea M. Mixed H2/Hœ control: a convex optimization approach[J]. Automatic Control, IEEE Transactions on, 1991, 36(7): 824-837.

[13] El Ghaoui L, Feron E, Balakrishnan V. Linear matrix inequalities in system and control theory[M]. Philadelphia: Society for industrial and applied mathematics, 1994. 